\title{
A randomized clinical trial: optimal strategies of paravertebral nerve block combined with general anesthesia for postoperative analgesia in patients undergoing lobectomy: a comparison of the effects of different approaches for serratus anterior plane block
}

\author{
Jianxu Er ${ }^{1}$, Jing Xia ${ }^{2}$, Ruifang Gao ${ }^{3}$, Yonghao Yu ${ }^{1}$ \\ ${ }^{1}$ Department of Anesthesiology, Tianjin Medical University General Hospital Tianjin Research Institute of Anesthesiology, Tianjin, China; \\ ${ }^{2}$ Department of Pain, Tianjin Port Hospital, Tianjin, China; ${ }^{3}$ Department of Anesthesiology, Tianjin Chest Hospital, Tianjin, China \\ Contributions: (I) Conception and design: J Er; (II) Administrative support: Y Yu; (III) Provision of study materials or patients: J Xia; (IV) Collection and \\ assembly of data: R Gao; (V) Data analysis and interpretation: J Er; (VI) Manuscript writing: All authors; (VII) Final approval of manuscript: All authors. \\ Correspondence to: Yonghao Yu. Department of Anesthesiology, Tianjin Medical University General Hospital Tianjin Research Institute of \\ Anesthesiology, Tianjin 300052, China. Email: yuyonghao@126.com.
}

Background: To observe the analgesic effect of different ultrasound-guided methods of serratus anterior
plane block (SAPB) after surgery in patients who have undergone thoracoscopic lobectomy with general
anesthesia combined with thoracic paravertebral nerve block.

Methods: A total of 120 patients aged 18-65 years old scheduled for video-assisted thoracoscopic surgery (VATS) were selected. Patients were randomly divided into 3 groups: patient-controlled intravenous analgesia (PCIA) group, serratus anterior plane block (SPB) group and continuous serratus anterior plane block (CSPB) group ( $\mathrm{n}=40$ each). All patients were treated with general anesthesia combined with doublepoint (T4, T7) thoracic paravertebral block. The SPB group received an ultrasound-guided single serratus plane block. The CSPB group underwent the same procedure as the SPB group, with an epidural catheter inserted. Both the PCIA and SPB groups received PCIA after surgery. Patients in the CSPB group were connected to a continuous serratus block automatic analgesia pump after surgery.

Results: There were no significant differences among the 3 groups in terms of the general condition. Compared with the PCIA group, the resting and exercise VAS pain scores at T2, T3, T4, and T5 , cortisol level at T1, T4 and T5 in SPB group and CSPB group were lower $(\mathrm{P}<0.05)$, the times of the first analgesia were significantly prolonged, and the times of pressing the PCA pump and opioid use were significantly less in the SPB and CSPB groups, and the CSPB group used no opioids $(\mathrm{P}<0.05)$, the SPB group and CSPB group had shorter times of the first postoperative activity, longer mobilization distance and the total number of days in hospital was significantly lower $(\mathrm{P}<0.05)$. Postoperative complications in the SPB and CSPB groups were significantly less and the CSPB group had an even lower incidence of postoperative complications $(\mathrm{P}<0.05)$. Compared with the SPB group, active VAS pain scores at T4 and T5 were higher in the CSPB group $(\mathrm{P}<0.05)$. Compared with the PCIA group, the total QoR-40 score at T6 was significantly higher in the SPB and CSPB groups, and compared with the SPB group, this data was higher in the CSPB group $(\mathrm{P}<0.05)$.

Conclusions: Single ultrasound-guided SAPB combined with PCIA can provide a better analgesic effect, improve the quality of early postoperative recovery, and accelerate ERAS.

Trial Registration: Chinese Clinical Trial Registry ChiCTR2000041350.

Keywords: Serratus anterior plane block (SAPB); enhanced recovery after surgery; multimodal analgesia (MMA); video-assisted thoracoscopic surgery (VATS)

Submitted Aug 06, 2021. Accepted for publication Nov 22, 2021.

doi: 10.21037/apm-21-2597

View this article at: https://dx.doi.org/10.21037/apm-21-2597 


\section{Introduction}

Lung cancer is the most common cause of cancer-related death at home and abroad (1). Lobectomy plus lymph node dissection is an effective method for the radical treatment of lung cancer (2). The current electronic thoracoscopyassisted minimally invasive lobectomy technology [videoassisted thoracoscopic surgery (VATS)], with its small injury, less bleeding, and faster recovery advantages, has gradually replaced traditional thoracotomy lung resection $(3,4)$, and is the most common surgical procedure for the treatment of lung cancer. However, studies have shown that patients who received VATS had moderate or even severe pain $(5,6)$, and postoperative pain often leads to increased pulmonary complications, which thus prolongs hospital stay, increases medical costs, and affects the rapid postoperative recovery [enhanced recovery after surgery (ERAS)] of patients. Pain after thoracotomy means that the surgical incision has healed and the pain at the incision site persists for more than 2 months or repeated attacks after thoracotomy. This kind of pain is the most serious pain problem after surgery. Its particularity is that it can lead to a variety of serious complications, and may also develop into pain syndrome after thoracotomy.

Therefore, effective control of pain is an important part of accelerated rehabilitation surgery. Research suggests that thoracic nerve block [thoracic paravertebral block (TPVB)], higher security, can provide similar effects to thoracic epidural analgesia (TEA) for thoracoscopic surgery analgesia (7). However, it has been found in clinical work that the application of TPVB for postoperative analgesia after VATS also has some disadvantages: the single action time of TPVB is limited, the continuous catheters can be displaced and prolapse, and the incidence of incomplete block range is high (8), which does not meet the requirements of perioperative whole analgesia. At present, multimodal analgesia (MMA) is advocated in clinical anesthesia, and intravenous analgesia combined with regional nerve block is widely favored by anesthesiologists. In recent years, muscle plane block [serratus anterior plane block (SAPB)] has emerged as a new technology of regional nerve block, with a simple operation and less complications, and the anterior lateral chest wall nerve block effect more completely, gradually widely (9). However, there is no clinical consensus on single SAPB analgesia or continuous SAPB analgesia, as well as which method is more effective and has fewer complications when applied to MMA after VATS. This study intends to undertake a preliminary discussion on this issue.

We present the following article in accordance with the CONSORT reporting checklist (available at https://dx.doi. org/10.21037/apm-21-2597).

\section{Methods}

This randomized controlled trial was designed according to the Consolidated Standards of Reporting Trials (CONSORT) 2010 statement. All procedures performed in this study involving human participants were in accordance with the Declaration of Helsinki (as revised in 2013). The study was approved by institutional board of Tianjin Chest Hospital (No.: 2021KY-018-01) and informed consent was taken from all the patients. This study was a threearm parallel study and allocation ratio was 1:1:1. A total of 120 patients, regardless of gender, with an American Society of Anesthesiologists (ASA) physical status classification of grade I-III, age 18-65 years old, and body mass index (BMI) 20-25, who planned to undergo thoracoscopic lobectomy in Tianjin Chest Hospital were selected. The exclusion criteria were as follows: allergy to local anesthetic drugs, severe abnormal coagulation function, systemic or puncture site infection, nerve injury, a history of longterm alcohol consumption, long-term use of psychotropic drugs and opioids, preoperative adjuvant chemotherapy or radiotherapy, not cooperative, involved in other clinical trials, and refusal to participate. Using the random number table method by computer, nurse generated the random allocation sequence, enrolled participants, and assigned participants to interventions, The generated random numbers and grouping contents were put into a sealed opaque envelope by the nurse and delivered to the anesthesiologist on the morning of the surgery. The patients were divided into the patientcontrolled intravenous analgesia group (PCIA group), the single serratus anterior plane block group (SPB group), and the continuous serratus anterior plane block group (CSPB group), with 40 cases in each group. Routine preoperative preparation including electrocardiogram (ECG), heart rate $(\mathrm{HR})$, oxygen saturation $\left(\mathrm{SpO}_{2}\right)$, and bispectral index (BIS) were monitored after entering the room, oxygen was inhaled by mask, and peripheral arterial and venous access was established. For the induction of anesthesia, midazolam $0.5-1.0 \mathrm{mg} / \mathrm{kg}$, sufentanil $0.5-1.0 \mu \mathrm{g} / \mathrm{kg}$, etomidate $0.2-0.3 \mathrm{mg} / \mathrm{kg}$, and cisatracurium $0.3 \mathrm{mg} / \mathrm{kg}$ were used. After adequate oxygenation and denitrification, doublelumen bronchial intubation was performed. Fiberoptic bronchoscopy was used to determine the location of the 
tracheal tube, which was then fixed, and the anesthesia machine was connected. The tidal volume was $6-8 \mathrm{~mL} / \mathrm{kg}$, frequency was $12-14 \mathrm{bpm}$, positive end-expiratory pressure (PEEP) was $4 \mathrm{mmHg}$, and oxygen concentration was $60-80 \%$. After intubation, the patient was changed to the lateral decubitus position on the operative side, and the T4 and T7 TPVB on the affected side was guided by ultrasound. The $22 \mathrm{G}$ puncture needle was inserted into the plane, and the corresponding paravertebral space was reached under direct ultrasound. A $10 \mathrm{~mL}$ dose of $0.4 \%$ ropivacaine was given at each point. For anesthesia maintenance, an intravenous target-controlled infusion (TCI) of propofol was used, the plasma target concentration was $1-2 \mu \mathrm{g} / \mathrm{mL}$, and cisatracurium $5-10 \mathrm{mg}$ was administered intermittently. Intraoperative blood pressure was maintained at 20\% above or below the baseline, with a BIS of 40-60 and $\mathrm{PETCO}_{2}$ $35-45 \mathrm{mmHg}$.

Before awakening after surgery, the patients were maintained in the position during surgery. After aseptic preparation of the skin with iodophor, the SPB group patients the ultra-sound probe was placed over the midclavicular region of the thoracic cage in the sagittal plane, and then the subcutaneous tissue, latissimus dorsi, serratus anterior, intercostal muscle, and pleura superficial to the fourth and fifth ribs in the midaxillary line were identified. The superficial SAPB was targeted to the interfascial plane between the serratus anterior muscle and the latissimus dorsi muscle. Once again, there was no blood and no gas, and the remaining $0.375 \%$ ropivacaine was injected slowly, with $15 \mathrm{~mL}$ in total. In the CSPB group, the serratus anterior muscle was punctured by the same method, and $15 \mathrm{~mL} 0.375 \%$ ropivacaine was injected into the serratus anterior muscle, and then the epidural catheter was inserted. The depth of the catheter was $5 \mathrm{~cm}$, and the position of the catheter was determined again by ultrasound after the skin was properly fixed. Patients in the 3 groups recovered after surgery, and the endotracheal tube was removed after reaching the indication of extubation. The analgesia pump was connected before returning to the postoperative care unit of thoracic surgery. Patients in the PCIA group and SPB group were connected to an intravenous controlled analgesia pump, and the formula was: sufentanil $2-3 \mu \mathrm{g} / \mathrm{kg}$ + butoranol $12 \mathrm{mg}+0.9 \%$ sodium chloride injection to $150 \mathrm{~mL}$, background dose was $2 \mathrm{~mL} / \mathrm{h}$, patient-controlled analgesia (PCA) dose was $2 \mathrm{~mL}$, and the locking time was $15 \mathrm{~min}$. The CSPB group was connected to a continuous serratus anterior block automatic analgesia pump, and the formula was: $0.5 \%$ ropivacaine normal saline $300 \mathrm{~mL}$, background infusion dose $6 \mathrm{~mL} / \mathrm{h}$, PCA dose $6 \mathrm{~mL}$, and locking time $45 \mathrm{~min}$. The duration of selfcontrolled analgesia in the 3 groups was $48 \mathrm{~h}$ after surgery. For example, when Visual Analogue Scale (VAS) score $>4$, the self-controlled analgesia pump could be pressed once. If the analgesia was ineffective, an intramuscular morphine injection of $10 \mathrm{mg}$ was administered for analgesia. Anesthesiologist was blinded after surgery, those assessing outcomes were completed by the ICU investigators.

The primary outcomes observed in this study included: (I) VAS scores at (T2), 6 h (T3), 12 h (T4), 24 h (T5) after intubation and $48 \mathrm{~h}$ (T6) after surgery; (II) the first postoperative analgesia time, the number of effective presses of the electronic analgesia pump $48 \mathrm{~h}$ after the operation, the total amount of opioids used in the electronic analgesia pump, and the number of times of postoperative analgesia relief. The following contents are secondary outcomes: The general condition of the patients (gender, age, IBM), the duration of the operation, and the amount of intraoperative blood loss; Arterial partial pressure of oxygen $\left(\mathrm{PaO}_{2}\right)$, lactic acid (LAC), blood glucose (GLU) before surgery (T1), and $12 \mathrm{~h}$ (T4), $24 \mathrm{~h}$ (T5) after surgery; In addition, postoperative adverse reactions, such as nausea, vomiting, and dizziness, were recorded. One day before surgery (T0) and $48 \mathrm{~h}$ after surgery (T6), patients' quality of recovery (QoR-40) scores was also recorded, along with the time of the first postoperative activity and the total length of hospitalization (days). The incidence of chronic pain was assessed and recorded by telephone follow-up 2 months after surgery.

The sample size was calculated based on our pilot study, in which the mean VAS at $24 \mathrm{~h}$ after surgery was 4.8 in the PCIA group, with an approximate standard deviation of 1.1. A 1.5-point decrease in the VAS score was considered clinically significant. For a study power of $80 \%$ and an $\alpha$ value of 0.05 , the required sample size per group was calculated to be 34 . Given an estimated dropout rate of $15 \%$, we recruited 40 patients (after applying the preoperative exclusion criteria) for each group.

This study was a double-blind trial, and patients and postoperative follow-up personnel were unaware of the patient groups. If severe adverse events were observed in participants during the study, physicians were expected to execute an emergency break and perform relevant treatments based on the situation.

\section{Statistical analysis}

SPSS19.0 software was used for statistical analysis. 


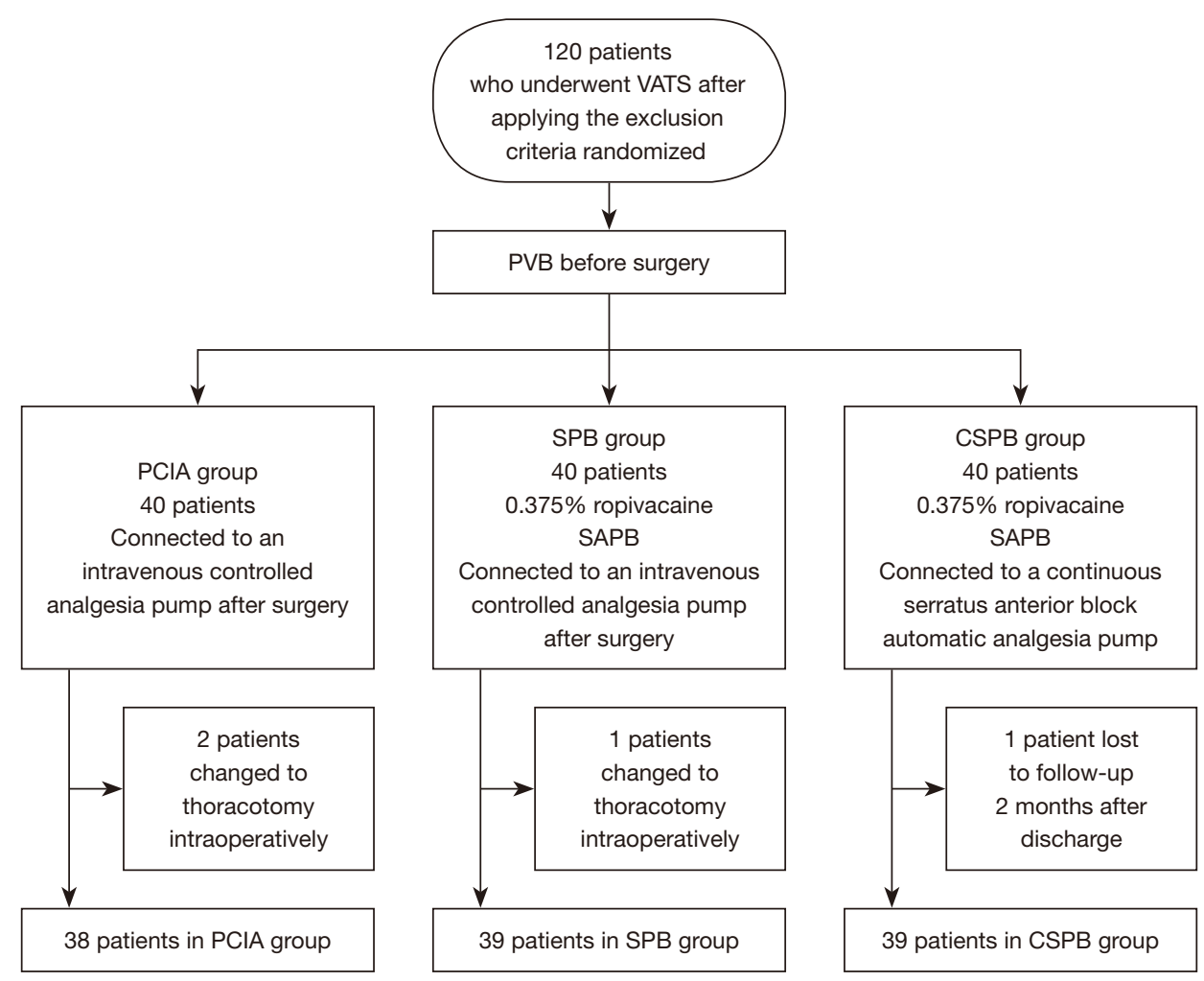

Figure 1 Participant enrollment. VATS, video-assisted thoracoscopic surgery; PVB, paravertebral block; PCIA, patient-controlled intravenous analgesia; SPB, serratus anterior plane block; SAPB, serratus anterior plane block; CSPB, continuous serratus anterior plane block.

Measurement data conforming to a normal distribution were expressed as mean \pm standard deviation $(\bar{x} \pm \mathbf{s})$, while measurement data with a skewed distribution were expressed as median and interquartile range (IQR). Oneway ANOVA was used for comparisons between groups, and the $t$-test was used for pair comparisons. Enumeration data were expressed as percentages, and the chi-square test was used. $\mathrm{P}<0.05$ was considered statistically significant.

\section{Results}

120 patients who underwent VATS after applying the exclusion criteria, 3 were changed to thoracotomy intraoperatively (2 in the PCIA group, 1 in the CSPB group), and 1 was lost to follow-up 2 months after discharge (SPB group). Finally, 116 patients completed the study from December 2020 to June 2021 (38 in the PCIA group, 39 in the SPB group, and 39 in the CSPB group), Figure 1. There was no significant difference in the general condition of the 3 groups $(\mathrm{P}>0.05)$, as shown in Table 1 .
Compared with the PCIA group, resting and exercise VAS scores were significantly decreased in the SPB group and CSPB group at T2-5 $(\mathrm{P}<0.05)$. Compared with the CSPB group, the VAS score during exercise in the SPB group was significantly decreased at T4 and T5 $(\mathrm{P}<0.05)$. At other time points, VAS scores at rest and exercise showed no significant differences among the 3 groups $(\mathrm{P}>0.05)$, as shown in Table 2.

Compared with the PCIA group, the first analgesia time in the SPB group and CSPB group was significantly longer, the effective press times of the analgesia pump within $48 \mathrm{~h}$ were significantly decreased, the number of times of postoperative analgesia was significantly decreased $(\mathrm{P}<0.05)$, and the consumption of sufentanil in the analgesia pump in the SPB group was significantly decreased $(\mathrm{P}<0.05)$. Compared with the SPB group, the consumption of sufentanil in the analgesia pump in the CSPB group was significantly decreased $(\mathrm{P}<0.05)$, however, there were no significant differences in the first analgesia time, the number of effective presses of the PCA pump, and the number of 
Table 1 Comparison of the general condition of the three groups

\begin{tabular}{lccc}
\hline Variables & PCIA group $(\mathrm{n}=38)$ & SPB group $(\mathrm{n}=39)$ & CSPB group $(\mathrm{n}=39)$ \\
\hline Sex $(\mathrm{M} / \mathrm{F})$ & $23 / 15$ & $21 / 18$ & $23 / 16$ \\
Age $($ year) & $52.36 \pm 10.87$ & $53.47 \pm 11.63$ & $56.34 \pm 11.64$ \\
BMI $\left(\mathrm{kg} / \mathrm{m}^{2}\right)$ & $25.65 \pm 4.21$ & $25.63 \pm 5.16$ & $25.96 \pm 5.33$ \\
Operation time $(\mathrm{min})$ & $127.52 \pm 6.68$ & $130.82 \pm 9.61$ & $129.78 \pm 7.82$ \\
Intraoperative blood loss $(\mathrm{mL})$ & $42.31 \pm 7.76$ & $40.63 \pm 10.21$ & $40.12 \pm 10.36$ \\
QoR-40 score at T0 & $193.87 \pm 4.33$ & $194.63 \pm 4.41$ & $194.70 \pm 4.65$
\end{tabular}

PCIA, patient-controlled intravenous analgesia; SPB, single serratus anterior plane block; CSPB, continuous serratus anterior plane block; BMI, body mass index.

Table 2 Comparison of VAS scores during rest and exercise at different postoperative time points in the three groups

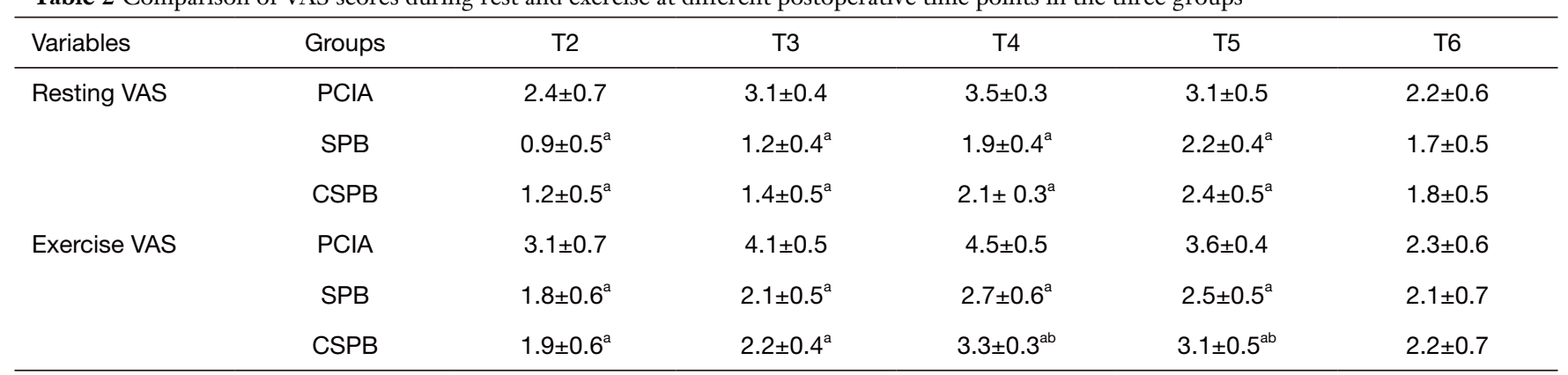

a , compared with the PCIA group, $\mathrm{P}<0.05 ;{ }^{\mathrm{b}}, \mathrm{P}<0.05$ compared with the SPB group. VAS, Visual Analogue Scale; PCIA, patient-controlled intravenous analgesia; SPB, single serratus anterior plane block; CSPB, continuous serratus anterior plane block.

Table 3 Comparison of other pain-related indicators among the three groups

\begin{tabular}{lcccc}
\hline Groups & $\begin{array}{c}\text { First analgesia time, } h, \\
\mathrm{M}(\mathrm{IQR})\end{array}$ & $\begin{array}{c}\text { Cumulative times of PCA, } \\
\mathrm{M}(\mathrm{IQR})\end{array}$ & $\begin{array}{c}\text { Cumulative dosage of } \\
\text { sufentanil }(\mu \mathrm{\mu g})\end{array}$ & $\begin{array}{c}\text { Rescue analgesia } \\
(\text { times })\end{array}$ \\
\hline PCIA group & $5(2.5)$ & $4(2.5)$ & $165.2 \pm 6.5$ & $0.85 \pm 0.75$ \\
SPB group & $10(7.0)^{\mathrm{a}}$ & $2(1.5)^{\mathrm{a}}$ & $124.4 \pm 3.2^{\mathrm{a}}$ & $0.05 \pm 0.22^{\mathrm{a}}$ \\
CSPB group & $8(10.0)^{\mathrm{a}}$ & $2(2.0)^{\mathrm{a}}$ & $0^{\mathrm{ab}}$ & $0.05 \pm 0.22^{\mathrm{a}}$ \\
\hline
\end{tabular}

a , compared with the PCIA group, $\mathrm{P}<0.05$; $^{\mathrm{b}}, \mathrm{P}<0.05$ compared with the SPB group. PCA, patient-controlled analgesia; $\mathrm{PCIA}$, patient-controlled intravenous analgesia; SPB, single serratus anterior plane block; CSPB, continuous serratus anterior plane block.

times of postoperative analgesia relief between the 2 groups $(\mathrm{P}>0.05)$, as shown in Table 3.

Blood gas analysis showed that the levels of Lac and Glu were increased at $\mathrm{T} 4$ and $\mathrm{T} 5$ compared with $\mathrm{T} 1(\mathrm{P}<0.05)$, and there was no significant difference in the change of $\mathrm{PaO}_{2}(\mathrm{P}>0.05)$. Compared with PCIA group, the level of plasma cortisol in SPB group and CSPB group decreased significantly at these time point. There were no significant differences in $\mathrm{PaO}_{2}$, Lac, and Glu among the 3 groups at each time point $(\mathrm{P}>0.05)$, as shown in Table 4.
Compared with the PCIA group, total QoR-40 scores, physical comfort, postoperative self-care ability, and pain scores in the SPB group and CSPB group were significantly increased at $48 \mathrm{~h}(\mathrm{~T} 6)$ after surgery $(\mathrm{P}<0.05)$. Compared with the SPB group, the total QoR-40 score and the score of physical comfort in the CSPB group increased at T6, while the pain score decreased $(\mathrm{P}<0.05)$, as shown in Table 5 .

Compared with the PCIA group, the incidence of nausea, vomiting and dizziness in the SPB group and CSPB group were significantly decreased $(\mathrm{P}<0.05)$. Compared with the 
Table 4 Comparison of $\mathrm{PaO}_{2}$, Lac, and Glu among the three groups

\begin{tabular}{|c|c|c|c|c|}
\hline Variables & Groups & $\mathrm{T} 1$ & $\mathrm{~T} 4$ & T5 \\
\hline \multirow{2}{*}{$\mathrm{PaO}_{2}(\mathrm{mmHg})$} & SPB & $93.38 \pm 2.91$ & $92.08 \pm 3.42$ & $92.72 \pm 2.35$ \\
\hline & CSPB & $93.21 \pm 2.78$ & $92.14 \pm 3.34$ & $92.82 \pm 2.19$ \\
\hline \multirow[t]{2}{*}{ Lac (mmol/L) } & PCIA & $1.45 \pm 0.22$ & $2.12 \pm 0.65^{\star}$ & $1.83 \pm 0.43^{\star}$ \\
\hline & CSPB & $1.37 \pm 0.47$ & $2.01 \pm 0.32^{*}$ & $1.78 \pm 0.44^{*}$ \\
\hline \multirow[t]{3}{*}{ Cortisol (nmol/L) } & PCIA & $678 \pm 22^{b}$ & $506 \pm 57^{b}$ & $548 \pm 47^{b}$ \\
\hline & SPB & $378 \pm 38^{a}$ & $386 \pm 39^{a}$ & $321 \pm 45^{\mathrm{a}}$ \\
\hline & CSPB & $366 \pm 37^{a}$ & $377 \pm 32^{\mathrm{a}}$ & $308 \pm 25^{\mathrm{a}}$ \\
\hline Glu (g/L) & CSPB & $6.15 \pm 1.21$ & $7.59 \pm 1.90^{*}$ & $7.09 \pm 1.56^{*}$ \\
\hline
\end{tabular}

*, compared with $\mathrm{T} 1, \mathrm{P}<0.05{ }^{a}$, compared with the $\mathrm{PCIA}$ group, $\mathrm{P}<0.05 ;{ }^{\mathrm{b}}, \mathrm{P}<0.05$ compared with the $\mathrm{SPB}$ group. $\mathrm{PaO}{ }_{2}$, partial arterial oxygen pressure; Lac, lactic acid level; Glu, blood glucose level; PCIA, patient-controlled intravenous analgesia; SPB, single serratus anterior plane block; CSPB, continuous serratus anterior plane block.

Table 5 Comparison of QoR-40 scores $48 \mathrm{~h}$ after surgery in the three groups

\begin{tabular}{|c|c|c|c|c|c|c|}
\hline Groups & Total & Emotional state & Physical comfort & Psychological support & Self-care ability & Pain \\
\hline SPB & $185.1 \pm 4.1^{\mathrm{a}}$ & $42.4 \pm 1.0$ & $53.8 \pm 1.7^{a}$ & $32.5 \pm 2.2$ & $24.0 \pm 0.8^{a}$ & $34.2 \pm 0.9^{\mathrm{a}}$ \\
\hline CSPB & $188.6 \pm 3.6^{\mathrm{ab}}$ & $42.5 \pm 1.8$ & $58.0 \pm 1.1^{\mathrm{ab}}$ & $31.5 \pm 1.7$ & $24.1 \pm 0.6^{a}$ & $32.1 \pm 0.7^{\mathrm{ab}}$ \\
\hline
\end{tabular}

${ }^{\mathrm{a}}$, compared with the PCIA group, $\mathrm{P}<0.05 ;{ }^{\mathrm{b}}$, $\mathrm{P}<0.05$ compared with the SPB group. PCIA, patient-controlled intravenous analgesia; SPB, single serratus anterior plane block; CSPB, continuous serratus anterior plane block.

Table 6 Comparison of the incidence of postoperative adverse reactions and postoperative chronic pain in the three groups [number of cases (percentage)]

\begin{tabular}{llll}
\hline Groups & Nausea and vomiting & Dizziness & Chronic pain \\
\hline PCIA & $12(31.6)$ & $10(26.3)$ & $7(18.4)$ \\
SPB & $7(17.9)^{\mathrm{a}}$ & $6(15.4)^{\mathrm{a}}$ & $6(15.3)$ \\
CSPB & $3(7.7)^{\mathrm{ab}}$ & $2(5.1)^{\mathrm{ab}}$ & $7(17.9)$ \\
\hline
\end{tabular}

${ }^{a}$, compared with the PCIA group, $\mathrm{P}<0.05 ;{ }^{\mathrm{b}}, \mathrm{P}<0.05$ compared with the SPB group. PCIA, patient-controlled intravenous analgesia; SPB, single serratus anterior plane block; CSPB, continuous serratus anterior plane block.

SPB group, the incidence of PONV and dizziness in the CSPB group was lower $(\mathrm{P}<0.05)$. There was no significant difference in the incidence of chronic pain among the 3 groups $(\mathrm{P}>0.05)$, as shown in Table 6 .

Compared with the PCIA group, the SPB group and
CSPB group had shorter times of the first postoperative activity, longer mobilization distance and the total number of days in hospital was significantly lower $(\mathrm{P}<0.05)$. Compared with the SPB group, the CSPB group had a longer time for the first postoperative activity and a longer 
Table 7 Comparison of postoperative recovery indexes in the three groups

\begin{tabular}{lccc}
\hline Groups & First postoperative activity $(\mathrm{h})$ & mobilization distance $(\mathrm{m})$ & Length of stay $($ day) \\
\hline PCIA & $17.5 \pm 2.1$ & $22.5 \pm 2.5$ & $12.5 \pm 2.6$ \\
SPB & $6.5 \pm 3.2^{\mathrm{a}}$ & $27.5 \pm 2.9^{\mathrm{a}}$ & $7.9 \pm 1.8^{\mathrm{a}}$ \\
CSPB & $10.3 \pm 2.9^{\mathrm{ab}}$ & $31.5 \pm 3.2^{\mathrm{a}}$ & $9.2 \pm 2.1^{\mathrm{ab}}$ \\
\hline
\end{tabular}

a, compared with the PCIA group, $\mathrm{P}<0.05$; $^{\mathrm{b}}, \mathrm{P}<0.05$ compared with the SPB group. PCIA, patient-controlled intravenous analgesia; SPB, single serratus anterior plane block; CSPB, continuous serratus anterior plane block.

total length of hospital stay $(\mathrm{P}>0.05)$, as shown in Table 7 .

\section{Discussion}

Moderate to severe pain occurs in $78 \%$ of patients after thoracic surgery (8). PCIA, mainly with opioid infusion, is associated with postoperative nausea and vomiting, respiratory depression, and intestinal obstruction (9). TEA has been known as the gold standard for thoracotomy postoperative analgesia. However, it is difficult to puncture the middle and high epidural block, with obvious intraoperative circulation fluctuation, postoperative urinary retention, nerve injury, and other complications (10) that limit its use. Studies have shown that TPVB, can provide similar effects to TEA in terms of thoracoscopic surgery analgesia (11). However, it has been found in clinical work that the application of TPVB in postoperative analgesia after VATS also has some disadvantages: the single time of action of TPVB is limited, the continuous catheters can be displaced and prolapse, and the incidence of incomplete block range is high (12), which does not meet the requirements of perioperative whole analgesia. New approaches to the TPVB for breast surgery have been proposed: the retrolaminar block (RLB) and the mid-point transverse process to pleura block (MTP block). In both cases, the local anesthetic is injected near the paravertebral space providing similar effect. A clear limitation of these techniques appears the inability to place a catheter for continuous postoperative infusion. At the current time, we do not recommend RLB and MTP blocks for pain management after VATS. Studies have shown that when TPVB is not feasible, both SAPB and ICNB can be regarded as the second choice (13).

Serratus anterior plane block and intercostal nerve block are attractive options for multimodal analgesia in patients undergoing thoracoscopic surgery. Intercostal nerve block can effectively block the nociceptive stimulation of intercostal nerve, but it needs multi-intercostal multi-point injection to give local anesthetic drugs, and the drug dosage is high, which is easy to produce drug poisoning reaction. Anterior serratus muscle plane block can block not only the lateral cortex of intercostal nerve, but also the long thoracic nerve, only single-point injection is needed and the range is more extensive. Ultrasound-guided SAPB involves injection of local anesthetic into the serratus anterior plane to block the lateral cutaneous branch of the T2-T9 intercostal nerve and provide anterolateral and partial posterior analgesia of the chest wall (9), which is suitable for postoperative analgesia after thoracoscopic surgery. Therefore, it is feasible and reliable to use intravenous anesthesia combined with TPVB for intraoperative analgesia, and to use SAPB or PCIA for postoperative analgesia.

In this study, it was found that the VAS scores of the SPB group and CSPB group were lower than those of the PCIA group at all time points except $48 \mathrm{~h}$ after surgery $(\mathrm{P}<0.05)$. Furthermore, the time of first postoperative activity was shortened and the total length of hospitalization was reduced, which confirmed that single and continuous SAPB as a component of postoperative analgesia of VATS could provide a good analgesic effect for patients in the early postoperative stage, and could accelerate patients' recovery. This is consistent with the research results of Magoon et al. (14) and Yang et al. (15). Hanley et al. (16) compared the effects of continuous SAPB and continuous TPVB on postoperative analgesia after VATS surgery, and the results showed that continuous SAPB, as part of the MMA program, did not have an inferior analgesic effect within $48 \mathrm{~h}$ after surgery, which confirmed the effectiveness of continuous SAPB in postoperative analgesia. However, Vig et al. (17) conducted continuous SAPB for 10 patients undergoing lateral thoracotomy, and 4 patients still needed additional pump injection for analgesia after surgery. Therefore, the authors believed that continuous SAPB had significant individual differences in alleviating postoperative pain after lateral thoracotomy. In this study, the VAS score of patients in the SPB group was lower than that in the 
CSPB group at 12 and $24 \mathrm{~h}$ after surgery $(\mathrm{P}<0.05)$, the total QoR-40 score in the SPB group was significantly higher than that in the CSPB group at $48 \mathrm{~h}$ after surgery, and the score of physical comfort was higher $(\mathrm{P}<0.05)$, which was consistent with the results of Vig et al. (17). It is suggested that continuous SAPB is inferior to single SAPB+PCIA in VATS surgery. The authors analyzed the significant differences in postoperative analgesic effects of continuous SAPB in different studies, which may be related to the following factors: (I) the superficial chest position, shallow and small fascial space, and different muscle mass, which may easily lead to the catheter falling out or the placement of the catheter is not ideal; (II) the surgical incision may destroy the fascial plane, thereby changing the distribution of drugs (17); (III) no consensus has been reached on the administration concentration and volume of SAPB, which affects the outcome. Subsequent studies are still needed to determine the optimal administration and catheterization methods of SAPB.

There are some limitations in this study: (I) this study is a single-center study with a small sample size. Postoperative follow-up was only performed 2 months after surgery, and longer follow-up was not conducted; (II) in this study, the internationally accepted VAS score was adopted for the evaluation of postoperative pain in the 3 groups of patients, but it is highly subjective. More objective indicators, such as the determination and comparison of inflammatory factors generated under pain stimulation, should be included.

In conclusion, ultrasonic-guided single SAPB combined with intravenous self-controlled analgesia, as a component of MMA after thoracoscopic lobotomy, has a good analgesic effect, fewer adverse reactions, and high patient comfort, which can improve the quality of early postoperative recovery and accelerate the perioperative recovery process of patients.

\section{Acknowledgments}

Funding: This work was supported by the Tianjin Health Commission science and technology talent training project (No. kj20072).

\section{Footnote}

Reporting Checklist: The authors have completed the CONSORT reporting checklist. Available at https://dx.doi. org/10.21037/apm-21-2597

Trial Protocol: Available at https://dx.doi.org/10.21037/apm-
$21-2597$

Data Sharing Statement: Available at https://dx.doi. org/10.21037/apm-21-2597

Conflicts of Interest: All authors have completed the ICMJE uniform disclosure form (available at https://dx.doi. org/10.21037/apm-21-2597). The authors have no conflicts of interest to declare.

Ethical Statement: The authors are accountable for all aspects of the work in ensuring that questions related to the accuracy or integrity of any part of the work are appropriately investigated and resolved. All procedures performed in this study involving human participants were in accordance with the Declaration of Helsinki (as revised in 2013). The study was approved by the Institutional Review Boards of Tianjin Chest Hospital (No.: 2021KY-018-01). Written informed consent was obtained from each patient and their guardian.

Open Access Statement: This is an Open Access article distributed in accordance with the Creative Commons Attribution-NonCommercial-NoDerivs 4.0 International License (CC BY-NC-ND 4.0), which permits the noncommercial replication and distribution of the article with the strict proviso that no changes or edits are made and the original work is properly cited (including links to both the formal publication through the relevant DOI and the license). See: https://creativecommons.org/licenses/by-nc-nd/4.0/.

\section{References}

1. Matsuoka K, Yamada T, Matsuoka T, et al. Video-assisted thoracoscopic surgery for lung cancer after induction therapy. Asian Cardiovasc Thorac Ann 2018;26:608-14.

2. Chen C, Ni Q, Shi Y, et al. Prognosis analysis of lobectomy and sublobar resection in patients $\geq 75$ years old with pathological stage I invasive lung adenocarcinoma of $\leq 3 \mathrm{~cm}$ : a propensity score matching-based analysis. Transl Cancer Res 2019;8:574-82.

3. Bowman JA, Utter GH. Electronic chest tube drainage devices and low suction following video-assisted thoracoscopic pulmonary lobectomy. J Thorac Dis 2019;11:1738-41.

4. Huang $\mathrm{L}$, Zheng J, Hu R, et al. Uniportal video-assisted thoracoscopic surgery for lung neoplasms with tracheal bronchus: a case report. Transl Cancer Res 2020;9:3721-4. 
5. Wang BY, Liu CY, Hsu PK, et al. Single-incision versus multiple-incision thoracoscopic lobectomy and segmentectomy: a propensity-matched analysis. Ann Surg 2015;261:793-9.

6. Khoshbin E, Al-Jilaihawi AN, Scott NB, et al. An audit of pain control pathways following video-assisted thoracoscopic surgery. Innovations (Phila) 2011;6:248-52.

7. Komatsu T, Kino A, Inoue M, et al. Paravertebral block for video-assisted thoracoscopic surgery: analgesic effectiveness and role in fast-track surgery. Int J Surg 2014;12:936-9.

8. Okajima H, Tanaka O, Ushio M, et al. Ultrasoundguided continuous thoracic paravertebral block provides comparable analgesia and fewer episodes of hypotension than continuous epidural block after lung surgery. J Anesth 2015;29:373-8.

9. Mayes J, Davison E, Panahi P, et al. An anatomical evaluation of the serratus anterior plane block. Anaesthesia 2016;71:1064-9.

10. Ahlbeck K. Opioids: a two-faced Janus. Curr Med Res Opin 2011;27:439-48.

11. Kaur RP, Anwar S. Serratus Anterior Plane Block Versus Thoracic Epidural Analgesia for Post-Thoracotomy Pain Relief. J Cardiothorac Vasc Anesth 2018;32:e11-2.

12. Piccioni F, Langer M, Fumagalli L, et al. Thoracic paravertebral anaesthesia for awake video-assisted

Cite this article as: Er J, Xia J, Gao R, Yu Y. A randomized clinical trial: optimal strategies of paravertebral nerve block combined with general anesthesia for postoperative analgesia in patients undergoing lobectomy: a comparison of the effects of different approaches for serratus anterior plane block. Ann Palliat Med 2021;10(11):11464-11472. doi: 10.21037/apm-212597 thoracoscopic surgery daily. Anaesthesia 2010;65:1221-4.

13. Piccioni F, Segat M, Falini S, et al. Enhanced recovery pathways in thoracic surgery from Italian VATS Group: perioperative analgesia protocols. J Thorac Dis 2018;10:S555-63.

14. Magoon R, Kaushal B, Chauhan S, et al. A randomised controlled comparison of serratus anterior plane, pectoral nerves and intercostal nerve block for post-thoracotomy analgesia in adult cardiac surgery. Indian J Anaesth 2020;64:1018-24.

15. Yang XL, Gu H, Hu JC, et al. Operation, Effectiveness, and Limitations of Continuous Serratus Anterior Plane Blocks for Thoracoscopic Surgery in Adults. J Pain Res 2020;13:2401-10.

16. Hanley C, Wall T, Bukowska I, et al. Ultrasound-guided continuous deep serratus anterior plane block versus continuous thoracic paravertebral block for perioperative analgesia in videoscopic-assisted thoracic surgery. Eur J Pain 2020;24:828-38.

17. Vig S, Bhan S, Ahuja D, et al. Serratus Anterior Plane Block for Post-Thoracotomy Analgesia: a Novel Technique for the Surgeon and Anaesthetist. Indian J Surg Oncol 2019;10:535-9.

(English Language Editor: C. Betlzar) 